\title{
Supplementing the maternal diet of rats with butyrate enhances mitochondrial biogenesis in the skeletal muscles of weaned offspring
}

\author{
Yanping Huang, Shixing Gao, Guo Jun, Ruqian Zhao and Xiaojing Yang* \\ Key Laboratory of Animal Physiology \& Biochemistry, Nanjing Agricultural University, Nanjing 210095, People's \\ Republic of China \\ (Submitted 14 April 2016 - Final revision received 21 November 2016 - Accepted 5 December 2016 - First published online 16 January 2017)
}

\section{Abstract}

The present study aimed to investigate the effects of maternal dietary butyrate supplementation on energy metabolism and mitochondrial biogenesis in offspring skeletal muscle and the possible mediating mechanisms. Virgin female rats were randomly assigned to either control or butyrate diets ( $1 \%$ butyrate sodium) throughout gestation and lactation. At the end of lactation (21 d), the offspring were killed by exsanguination from the abdominal aorta under anaesthesia. The results showed that maternal butyrate supplementation throughout gestation and lactation did not affect offspring body weight. However, the protein expressions of G-protein-coupled receptors (GPR) 43 and 41 were significantly enhanced in offspring skeletal muscle of the maternal butyrate-supplemented group. The ATP content, most of mitochondrial DNA-encoded gene expressions, the cytochrome c oxidase subunit 1 and 4 protein contents and the mitochondrial DNA copy number were significantly higher in the butyrate group than in the control group. Meanwhile, the protein expressions of type 1 myosin heavy chain, mitochondrial transcription factor A, PPAR-coactivator- $1 \alpha$ (PGC- $1 \alpha$ ) and uncoupling protein 3 were significantly increased in the gastrocnemius muscle of the treatment group compared with the control group. These results indicate for the first time that maternal butyrate supplementation during the gestation and lactation periods influenced energy metabolism and mitochondrial biogenesis through the GPR and PGC- $1 \alpha$ pathways in offspring skeletal muscle at weaning.

\section{Key words: Maternal butyrate: Skeletal muscles: ATP: Mitochondrial biogenesis: Rats}

Dietary fibre is deficient in the diets of modern people. The lack of dietary fibre is viewed as one of the main reason for the current increasing rates of metabolic diseases. Normally, dietary fibre is digested and absorbed in the gastrointestinal tract by complete or partial fermentation to SCFA such as butyric $\operatorname{acid}^{(1,2)}$. Considering the fibre effect on the diet of mothers during gestation and lactation, previous studies indicate that a high-fat, Western-style maternal diet, with additional dietary fibre, has a lower risk for developing CHD, hypertension, diabetes, obesity and other metabolic diseases in the offspring ${ }^{(3,4)}$. However, the underlying mechanism is still largely unclear.

In addition to being the energy source for ruminants and monogastric animals ${ }^{(5,6)}$, most previous studies suggest that butyrate, a kind of SCFA, might mediate the effects of gut microbiota and nutrition on immunity, metabolism, as well as the pathogenesis of obesity, diabetes mellitus and inflammatory bowel disease ${ }^{(7-10)}$. Previous studies have suggested that butyrate supplementation in high-fat diets could improve mitochondrial function by promoting a skeletal muscle type 1 fibre phenotype and physiology ${ }^{(10,11)}$. A few studies have demonstrated that maternal butyrate supplementation can improve offspring growth and antioxidant capacity ${ }^{(12,13)}$. However, whether maternal butyrate supplementation has an influence on energy metabolism and mitochondrial function in offspring skeletal muscle is still unknown.

Mitochondrial function has an important role in maintaining energy homoeostasis in skeletal muscle by balancing ATP generation and expenditure at the cellular level ${ }^{(11,14)}$. The functions of the skeletal muscle mostly depend on the highly efficient process of mitochondrial oxidative phosphorylation system (OXPHOS). Previous studies have demonstrated that the enhanced mitochondrial biogenesis could increase OXPHOS with ATP-generating capacity ${ }^{(15,16)}$. Mitochondrial biosynthesis is initiated with increased transcription of both nuclear and mitochondrial DNA (mtDNA; thirteen coding genes). The PPAR $\gamma$-coactivator (PGC-1 $\alpha$ ) family of proteins has been identified as the main family of transcriptional co-activators involved in the induction of mitochondrial biogenesis ${ }^{(17)}$. In particular, PGC- $1 \alpha$ has been reported to promote mitochondrial biogenesis and fibre-type transformation in skeletal muscle cells ${ }^{(18,19)}$. In addition, cAMP (cyclic adenosine monophosphate) response element-binding protein (CREB) induces PGC- $1 \alpha$ to regulate mitochondrial

Abbreviations: COX1, cytochrome c oxidase subunit 1; CREB, cAMP response element-binding protein; GPR, G-protein-coupled receptor; mtDNA, mitochondrial DNA; MYHC, myosin heavy chain; p-CREB, anti-phospho-ser ${ }^{133}$ CREB; TFAM, mitochondrial transcription factor A; UCP, uncoupling protein 3.

* Corresponding author: X. Yang, fax +86 258439 8669, email yangxj@njau.edu.cn 
biogenesis ${ }^{(20)}$. It has been shown that butyrate can influence body metabolism by activation of the endogenous G-protein-coupled receptors (GPR) 41 and $43^{(21,22)}$. A previous study demonstrated that SCFA-activated GPR43-stimulated mitochondrial biogenesis in brown adipocytes ${ }^{(23)}$. If maternal butyrate supplementation influences offspring skeletal muscle energy metabolism, the possible underlying mechanisms need further investigation.

Accordingly, in the present study, we investigated the effects of maternal butyrate supplementation during gestation and lactation on offspring energy metabolism and mitochondrial biogenesis in gastrocnemius muscle at weaning. The results provide important insights into the effects of maternal butyrate supplementation on offspring skeletal muscle metabolism.

\section{Methods}

\section{Experimental design and sampling}

In total, twenty-eight, virgin, female Sprague-Dawley rats were purchased from the Laboratory Animal Center, University of Jiangsu, and housed in individual cages under temperature- and humidity-controlled conditions with a $12 \mathrm{~h}$ light- $12 \mathrm{~h}$ dark cycle. After $7 \mathrm{~d}$ of acclimatisation, rats were mated, and conception was confirmed by the presence of spermatozoids in the vaginal wash. Once conception was confirmed, females were randomly assigned to either a control or a butyrate diet ( $1 \%$ butyrate sodium), with water provided ad libitum. The control diet was prepared following the recommendations of the American Institute of Nutrition for rodent growth (AIN-93G) (Table 1).

After delivering, the litter size and litter weight were recorded. The litters were adjusted to eight animals for each dam, maintaining the sex ratio as close as possible to 1:1. During lactation, dams continued to consume their assigned experimental diet. At the end of lactation ( $21 \mathrm{~d}$ ), they were killed by exsanguination from the abdominal aorta under anaesthesia with pentobarbital sodium. Blood samples of both mothers and offspring were collected, and serum was obtained by centrifugation at $3500 \mathrm{~g}$ for $10 \mathrm{~min}$. The gastrocnemius muscles of offspring were excised, weighed, snap-frozen in liquid $\mathrm{N}_{2}$ and immediately stored at $-80^{\circ} \mathrm{C}$ until analysis.

All animal procedures were approved by the Institutional Animal Care and Use Committee of Nanjing Agricultural

Table 1. Compositions of the experimental diets

\begin{tabular}{lcc}
\hline Ingredients (\%) & Control & Butyrate \\
\hline Maize & 47 & 46 \\
Wheat middlings & 24 & 24 \\
Soyabean meal & 10 & 10 \\
Fishmeal & 4 & 4 \\
Chicken meal & 5 & 5 \\
Premix & 4 & 4 \\
Salad oil & 1 & 1 \\
Limestone & 1 & 1 \\
Butyrate & 0 & 1 \\
Alfalfa & 4 & 4 \\
Total & 100 & 100 \\
Protein & 22 & 22 \\
Fat & $6 \cdot 5$ & 6.5 \\
Gross energy $(\mathrm{kJ} / \mathrm{g})$ & 17 & 17 \\
\hline
\end{tabular}

University. The protocol of this study was reviewed and approved with the project number 31572482. All experimental protocols strictly followed the 'Guidelines on Ethical Treatment of Experimental Animals' (2006) no. 398 set out by the Ministry of Science and Technology, China, and the Regulation regarding the Management and Treatment of Experimental Animals' (2008) no. 45 set out by the Jiangsu Provincial People's Government.

\section{Biochemical analysis}

Butyrate levels in the serum of dams were determined by GC. The extraction and gas chromatographic analysis procedures were carried out as described by Zhao et $a l^{(24)}$ with some modifications. In brief, chromatographic analysis was carried out using a Shimadzu GC-2010 Plus system (Shimadzu) equipped with a flame-ionisation detector (FID). A fused-silica capillary column with a free-fatty acid phase (DB-FFAP 125-3237; J\&W Scientific, Agilent Technologies Inc.) of $30 \mathrm{~m} \times 0.53-\mathrm{mm}$ I.D. (inner diameter) coated with a $0 \cdot 50-\mu \mathrm{m}$-thickness film was used. He was supplied as the carrier gas at a flow rate of $14.4 \mathrm{ml} / \mathrm{min}$. The initial oven temperature was $100^{\circ} \mathrm{C}$; it was maintained at the same level for $0.5 \mathrm{~min}$ and then raised to $180^{\circ} \mathrm{C}$ by $8^{\circ} \mathrm{C} / \mathrm{min}$ and held at the same level $1.0 \mathrm{~min}$; further, the temperature was increased to $200^{\circ} \mathrm{C}$ by $20^{\circ} \mathrm{C} / \mathrm{min}$ and finally held at $200^{\circ} \mathrm{C}$ for $5 \mathrm{~min}$. Glass wool (Supelco) was inserted in the glass liner of the splitless injection port. The temperatures of the FID and the injection port were 240 and $200^{\circ} \mathrm{C}$, respectively. The flow rates of $\mathrm{H}_{2}$, air and $\mathrm{N}$ as makeup gases were 30,300 and $20 \mathrm{ml} / \mathrm{min}$, respectively. The injected sample volume for GC analysis was $1 \mu \mathrm{l}$, and the running time for each analysis was $17.5 \mathrm{~min}$.

\section{RNA isolation and real-time PCR for mRNA quantification}

Total RNA was isolated from gastrocnemius muscle tissue with TRIzol reagent (Invitrogen Life Technologies), and reverse transcribed with the PrimeScript 1st Strand cDNA Synthesis Kit (RR048A; Takara) according to the manufacturer's instructions. Diluted complementary DNA $(2 \mu \mathrm{l}, 1: 25)$ was used in each real-time PCR assay with Mx3000P (Stratagene). All primers (Table 2) were synthesised by Generay Biotech. $\beta$-Actin was chosen as a reference gene as it is not affected by maternal butyrate supplementation.

\section{Western blotting analysis}

Total cellular proteins were extracted from $100 \mathrm{mg}$ of frozen gastrocnemius muscle tissue samples as previously described ${ }^{(25)}$. Nuclear protein extracts were used to detect the expression of PGC- $1 \alpha$. Protein concentration was measured with a Pierce BCA Protein Assay kit (no. 23225; Thermo Scientific). Western blot analysis of the target proteins was carried out according to the protocols provided by the manufacturer. The sources of primary antibodies used in the Western blot analysis are as follows: anti-GPR41 (no. sc-98332; Santa Cruz Biotechnology), anti-GPR43 (no. sc-32906; Santa Cruz Biotechnology), anti-mitochondrial transcription factor A (TFAM, no. ab176558; Abcam), antiMYHC1 (no. 05716; Millipore), anti-cytochrome c oxidase subunit 1 (COX1; no. BS1636; Bioworld Technology), anti-COX4 
Table 2. Primer sequences for quantitative RT-PCR of mRNA

\begin{tabular}{|c|c|c|c|}
\hline Gene name & Primer sequence $\left(5^{\prime}-3^{\prime}\right)$ & Accession no. & Product length (bp) \\
\hline $\operatorname{cox} 1$ & $\begin{array}{l}\text { F: AGTATTAGCAGCAGGTATCAC } \\
\text { R: GCCGAAGAATCAGAATAGGT }\end{array}$ & NC_001665.2 & 118 \\
\hline COX3 & $\begin{array}{l}\text { F: CCGTGAAGGAACATACCAA } \\
\text { R: TGATGCTAAGAGGACTGATG }\end{array}$ & NC_001665.2 & 235 \\
\hline CYTB & $\begin{array}{l}\text { F: ACTTCGGTTCTCTACTAGGA } \\
\text { R: TGGAGGAATAGGCAGATGA }\end{array}$ & NC_001665.2 & 196 \\
\hline ND1 & $\begin{array}{l}\text { F: GCCTGACCAATAGCCATAA } \\
\text { R: GCCTGAGACTAATTCTGATTC }\end{array}$ & NC_001665.2 & 99 \\
\hline ND2 & $\begin{array}{l}\text { F: CTAACCATAGCATCCATCATC } \\
\text { R: GTCGTGTATAGAAGAATAGGC }\end{array}$ & NC_001665.2 & 166 \\
\hline ND3 & $\begin{array}{l}\text { F: AACATCACTGCCCAAGC } \\
\text { R: CTTCCCACCAGTCACTTACA }\end{array}$ & NC_001665.2 & 93 \\
\hline ND4 & $\begin{array}{l}\text { F: CCACTAATAATGCTCGCTAG } \\
\text { R: CGGAGAATGTTATGATGAGT }\end{array}$ & NC_001665.2 & 115 \\
\hline ND4L & $\begin{array}{l}\text { F: CTCCATAATCTCCATAACCATC } \\
\text { R: CGTAGTCTGTTCCGTAAGTA }\end{array}$ & NC_001665.2 & 113 \\
\hline ND5 & $\begin{array}{l}\text { F: CTCATCAGTAAGCCATATAGC } \\
\text { R: TTCGTTCGTAGTTGGTGTT }\end{array}$ & NC_001665.2 & 146 \\
\hline ATP6 & $\begin{array}{l}\text { F: GGCTCAACCAACCTTCTA } \\
\text { R: TTACGGCTCCTGCTCATA }\end{array}$ & NC_001665.2 & 103 \\
\hline ATP8 & $\begin{array}{l}\text { F: CCACAACTAGACACATCCA } \\
\text { R: GGGTAATGAAAGAGGCAAAT }\end{array}$ & NC_001665.2 & 192 \\
\hline MYHC1 & $\begin{array}{l}\text { F: GAGGAGAGGGCGGACATT } \\
\text { R: ACTCTTCATTCAGGCCCTTG }\end{array}$ & NM_017240 & 181 \\
\hline MYHC2A & $\begin{array}{l}\text { F: GTGTCACTGTCAACCCCTAC } \\
\text { R: CTCCCGGTCAGTTAGCAT }\end{array}$ & NM_001135157 & 186 \\
\hline MYHC2X & $\begin{array}{l}\text { F: CCTGCAGCTCCAAGTTCAGT } \\
\text { R: ATCAGCTGGTCGCATCTTTC }\end{array}$ & NM_001135158 & 196 \\
\hline MYHC2B & $\begin{array}{l}\text { F: TGCCTCCTTCTTCATCTGGT } \\
\text { R: AGCAGCCTCCCCAAAAAC }\end{array}$ & NM_019325 & 99 \\
\hline Myogenin & $\begin{array}{l}\text { F: CCACCGTCCATTCACAT } \\
\text { R: GAGGGGCATTAACAAGG }\end{array}$ & NM_017115 & 129 \\
\hline Myoglobin & $\begin{array}{l}\text { F: AGAAGAGGGGAGCATAGTG } \\
\text { R: CTGGTGACTTGCATTTGG }\end{array}$ & NM_021588 & 220 \\
\hline MYOD1 & $\begin{array}{l}\text { F: CTGAGCAAAGTGAACGAGG } \\
\text { R: GCAGACCTTCAATGTAGCG }\end{array}$ & NM_176079 & 118 \\
\hline mTOR & $\begin{array}{l}\text { F: GCAGATTTGCCAACTACC } \\
\text { R: TCTCCGGCCCTCATTTCG }\end{array}$ & NM_019906 & 207 \\
\hline$\beta$-Actin & $\begin{array}{l}\text { F: GGGAAATCGTGCGTGAC } \\
\text { R: ACCCAGGAAGGAAGGCT }\end{array}$ & NM_031144 & 188 \\
\hline
\end{tabular}

(no. AP0705; Bioworld Technology), anti-phospho-ser ${ }^{133}$ CREB (p-CREB, no. BS6345; Bioworld Technology), anti-CREB (no. BS6230; Bioworld Technology), anti-PGC-1 $\alpha$ (no. sc-13067; Santa Cruz Biotechnology) and anti-uncoupling protein 3 (UCP3, no. BS2849; Bioworld Technology). Lamin A/C (no. BS3774; Bioworld Technology) was used to verify the purity of nuclear proteins. $\beta$-Actin (no. AP0060; Bioworld Technology), GAPDH (glyceraldehyde 3-phosphate dehydrogenase; MB001H; Bioworld Technology) and $\alpha$-tubulin (no. BS1699; Bioworld Technology) were used to verify total cellular proteins.

\section{Determination of $A M P, A D P, A T P$ and NAD concentrations in gastrocnemius muscle tissue}

The concentrations of AMP (adenosine monophosphate), ADP, ATP and NAD (aldehyde dehydrogenase) in gastrocnemius muscle were determined according to a previous study ${ }^{(26)}$. Concentrations were determined by HPLC with a reverse-phase column (C18, $5 \mu \mathrm{m}, 250 \times 4.6 \mathrm{~mm}$; Dikma Technologies Inc.).
Next, using a dual $\lambda$ absorbance detector (Waters Corporation), the detection wavelength of the HPLC was found to be $260 \mathrm{~nm}$. The mobile phase contained phosphate buffer $(50-\mathrm{mmol} / \mathrm{l}$ potassium dihydrogen orthophosphate, 50-mmol/1 dipotassium hydrogen orthophosphate, $\mathrm{pH}=5 \cdot 4$ ), 2.3-mm tetrabutylammonium hydrogen sulphate and $1 \%$ acetonitrile. The adenine nucleotides were eluted after $30 \mathrm{~min}$ of isocratic elution at a flow rate of $1 \mathrm{ml} / \mathrm{min}$. Energy charge (EC) was calculated using the following formula: $\mathrm{EC}=([\mathrm{ATP}]+1 / 2[\mathrm{ADP}]) /([\mathrm{ATP}]+[\mathrm{ADP}]+[\mathrm{AMP}])$.

\section{Determination of mitochondrial DNA copy number in gastrocnemius muscle tissue}

mtDNA copy number was measured in skeletal muscle of weaning offspring. In brief, total genomic DNA was isolated from skeletal muscle, and the mtDNA copy number was determined using real-time PCR as previously described ${ }^{(27)}$. Primers specific for the coding region of mtDNA (ATP synthase F0 subunit 8 (ATP8)) were used for the quantification of the 
mtDNA copy number, whereas primers specific for the $18 \mathrm{~S}$ nuclear gene were used for standardisation. The relative mtDNA copy number was calculated using the $2^{-\Delta \Delta C_{t}}$ method.

\section{Statistical analysis}

Numerical data are expressed as mean values with their standard errors. All statistical analyses were performed using Statistical Program for Social Sciences (SPSS) software 18.0 for Windows (SPSS Inc.). The $2^{-\Delta \Delta C_{t}}$ method was used to analyse the real-time PCR data. The differences were analysed using independent $t$ test with SPSS 18.0 for Windows. Differences were considered significant at $P<0.05$.

\section{Results}

\section{Performance of mother and offspring and serum butyrate content of dams}

Food intake of dams per week during gestation and lactation in the butyrate group did not change significantly compared with the control group (223.69 (SE 5.74) v. 224.89 (sE 6.16) g, $P>0.05$ ). Body weight of dams showed no significant difference between the butyrate group and the control group ${ }^{(28)}$. Maternal butyrate supplementation demonstrated a trend towards increasing litter weight, litter size $\mathrm{e}^{(28)}$ and the ratio of muscle weight:body weight (7.27 (sE 0.16) v. 6.79 (SE 0.19) g, $P<0 \cdot 1$ ) as well as enhanced muscle weight of the offspring (0.54 (SE 0.01) v. 0.48 (SE 0.02) g, $P<0.05)$ compared with the control group. The average weight of newborns (7.04 (SE 0.14) $v .7 \cdot 18$ (SE 0.12) g, $P>0.05)$ and the body weight of weaning rats ${ }^{(28)}$ showed no significant differences between the two groups.

Butyrate levels in the serum of supplemented dams were significantly higher compared with the control group (Fig. 1(a)). Our previous study has been reported that serum concentrations of total cholesterol and TGA in dams and weaned offspring did not change with butyrate supplementation ${ }^{(28)}$.

\section{Protein levels of G-protein-coupled receptor 43 and 41 in} gastrocnemius muscle

The expressions of GPR 43 and 41 proteins were determined in gastrocnemius muscle. Both GPR43 and GPR41 protein levels were significantly higher $(P<0.05)$ in offspring gastrocnemius muscle in the treated group compared with the control group (Fig. 1(b) and (c)).

\section{Contents of ATP, ADP, AMP and NAD in gastrocnemius muscle}

Gastrocnemius muscle ATP concentration was significantly higher $(P<0.05)$ in the butyrate group compared with the control group. However, the concentrations of ADP, AMP and NAD exhibited no obvious differences between the control and butyrate groups (Fig. 2(a)). EC of skeletal muscle in the butyrate group was higher $(P<0 \cdot 01)$ than the control group (Fig. 2(b)).
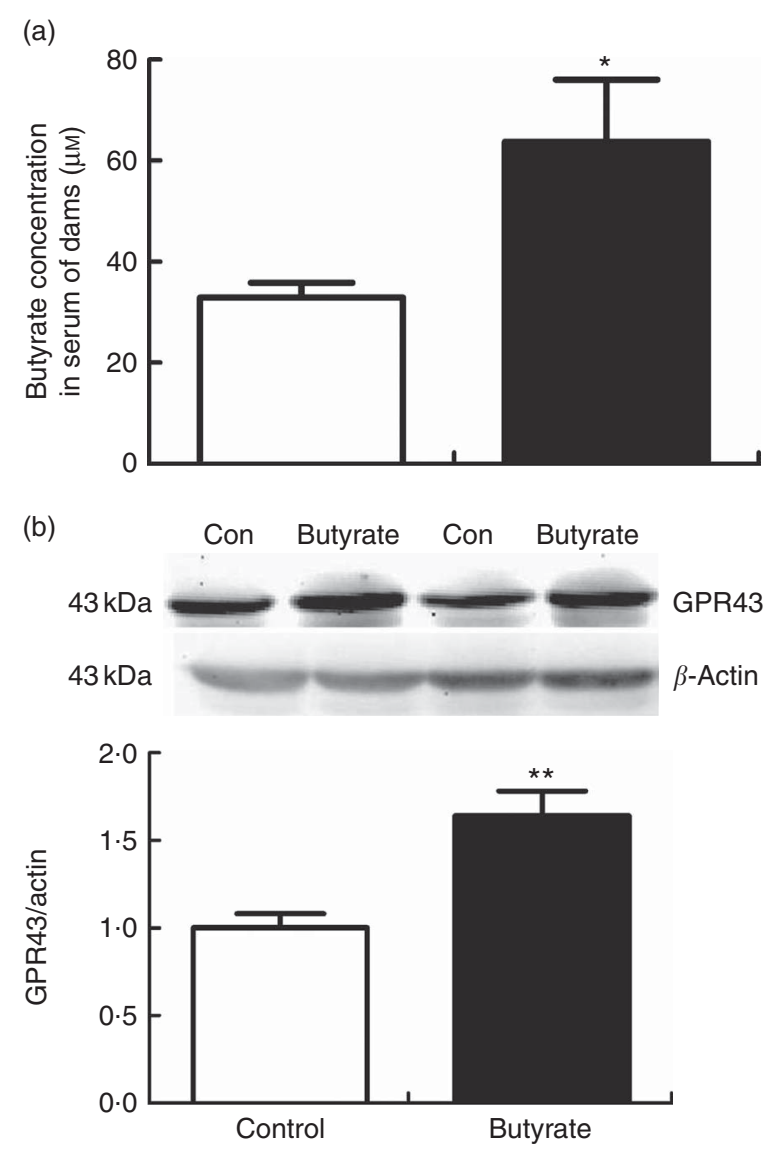

(c) Con Butyrate Con Butyrate
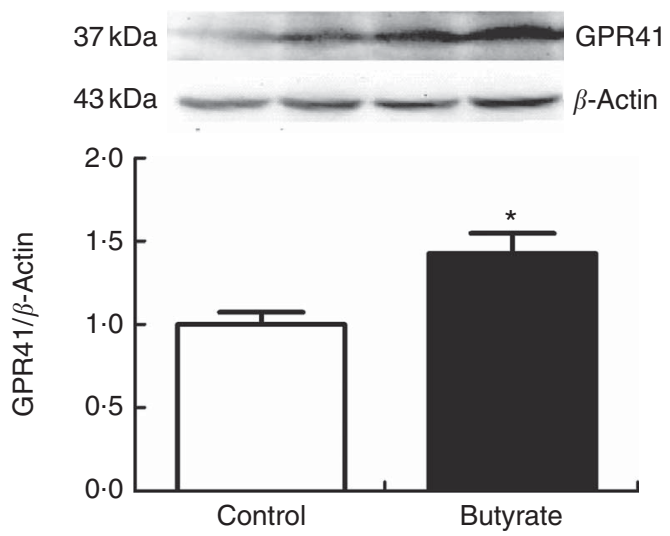

Fig. 1. Butyrate concentration in the serum of dams after weaning (a: $\square$, control; $\square$, butyrate); maternal butyrate supplementation throughout gestation and lactation increased G-protein-coupled receptors (GPR) 43 (b) and GPR41 (c) protein expressions in gastrocnemius muscle of weaning rats ( $n 7$ per group). $\beta$-Actin was used as the internal standard for Western blotting. Values are means with their standard errors. Con, control. ${ }^{*} P<0.05$, ${ }^{\star \star} P<0.01$, compared with control.

\section{Mitochondrial DNA-encoded mRNA expressions, cytochrome c oxidase protein expression and mitochondrial DNA copy number in gastrocnemius muscle}

The mRNA abundance of twelve genes involved in gastrocnemius muscle OXPHOS was assessed. Among these genes, 
(a)

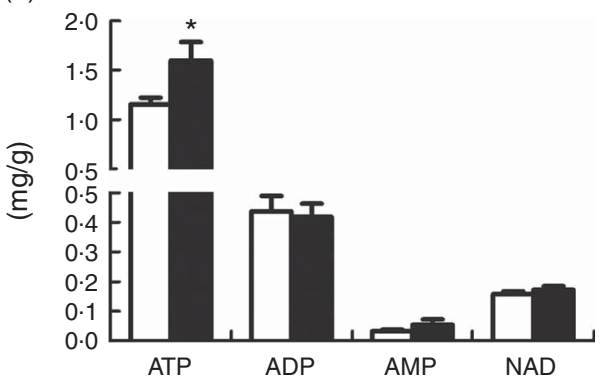

(c)

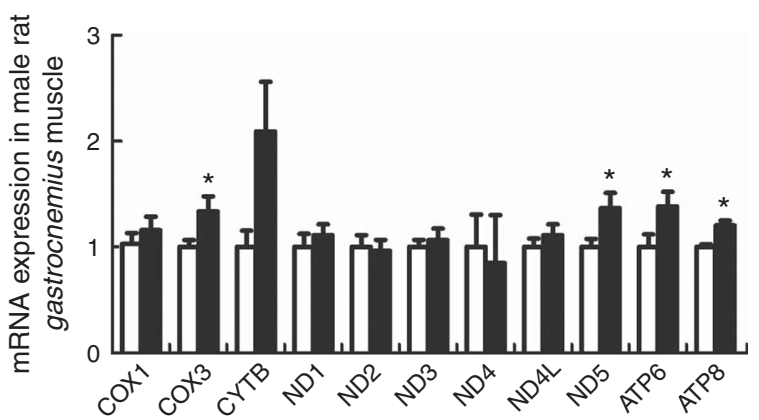

(e)
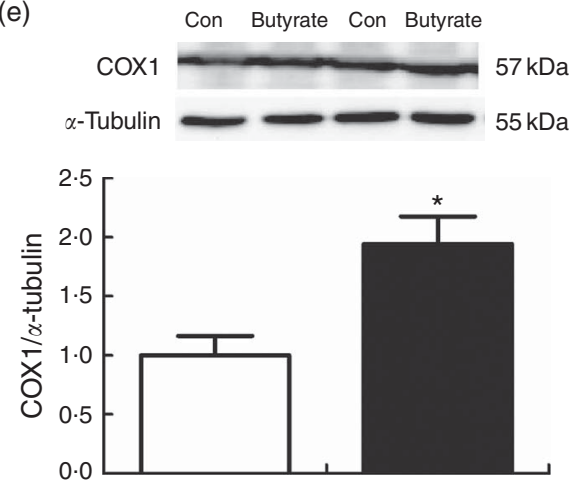

(b)

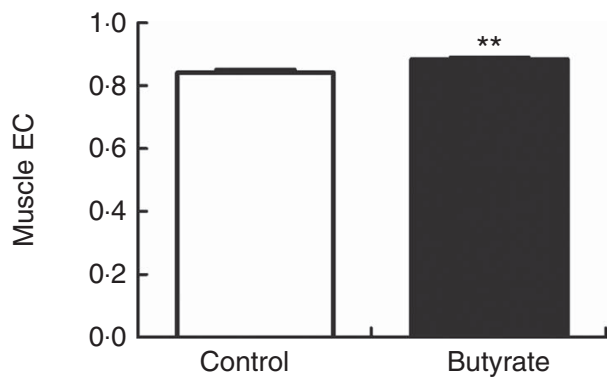

(d)
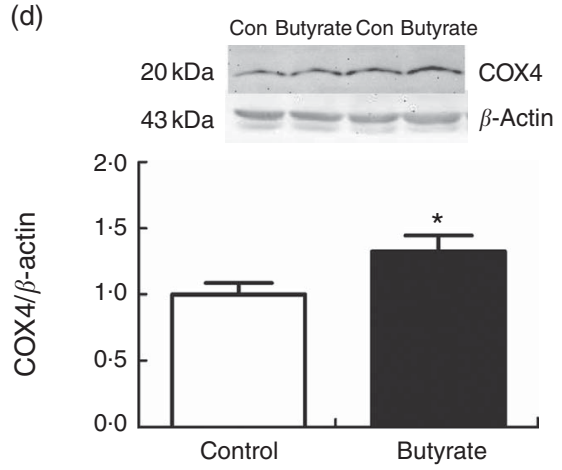

(f)

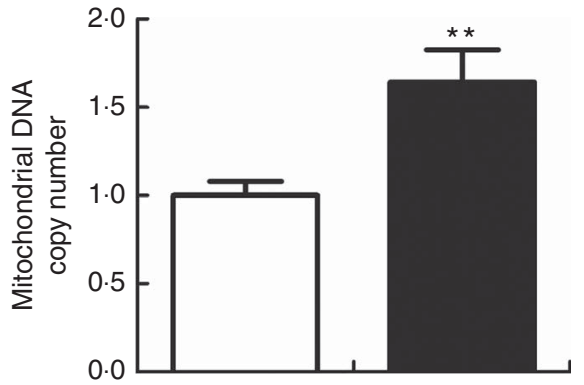

Fig. 2. Effect of maternal butyrate supplementation throughout gestation and lactation on gastrocnemius muscle ATP, ADP, AMP, NAD contents (a) and muscle energy charge (EC) (b) in weaning rats ( $n 5$ per group); mitochondrial DNA-encoded mRNA expression (c) ( $n 8$ per group) and cytochrome coxidase (COX) protein expression (d, e) ( $n$ per group) in gastrocnemius muscle; mitochondrial DNA copy number in gastrocnemius muscle in weaning rats (f); EC was calculated using the following formula: $E C=([A T P]+1 / 2[A D P]) /([A T P]+[A D P]+[A M P]) . \beta$-Actin and $\alpha$-tubulin were used as internal standards. Values are means with their standard errors. Con, control; COX1, cytochrome $c$ oxidase subunit 1; COX3,cytochrome $c$ oxidase subunit 3, CYTB, cytochrome b; ND1, NADH dehydrogenase subunit 1; ND2, NADH dehydrogenase subunit 2; ND3, NADH dehydrogenase subunit 3; ND4, NADH dehydrogenase subunit 4; ND4L, NADH dehydrogenase subunit 4L; ND5, NADH dehydrogenase subunit 5; ATP6: ATP synthase F0 subunit 6; ATP8, ATP synthase F0 subunit 8. a, c, e, f: $\square$, Control; $\boldsymbol{\square}$, butyrate. ${ }^{\star} P<0.05,{ }^{\star \star} P<0.01$, compared with control.

COX3, NADH dehydrogenase subunit 5 (ND5), ATP synthase F0 subunit 6 (ATP6) and ATP8 were significantly up-regulated in gastrocnemius muscle of the butyrate group compared with the control group $(P<0.05)$ (Fig. 2(c)). Consistent with the abundance of the aforementioned mRNA, the protein expressions of mitochondrial COX1 and COX4 were significantly increased in weaning rats born to butyrate-supplemented mothers compared with the control group $(P<0.05)$ (Fig. $2(d)$ and (e)). mtDNA copy number was also increased significantly in offspring skeletal muscle with maternal butyrate supplementation $(P<0 \cdot 01)$ (Fig. 2(f)).

\section{Energy metabolism-related protein content in gastrocnemius muscle}

Maternal butyrate supplementation significantly increased the protein expression of TFAM in gastrocnemius muscle of offspring rats (Fig. 3(a)). Maternal butyrate supplementation significantly increased the level of total CREB and p-CREB $(P<0.05)$, although the ratio did not change in gastrocnemius muscle of offspring rats (Fig. 3(b)). Compared with the control group, the levels of total PGC- $1 \alpha$, nuclear PGC- $1 \alpha$ and UCP3 $(P<0.05)$ proteins in weaning rats of the treated 
(a)

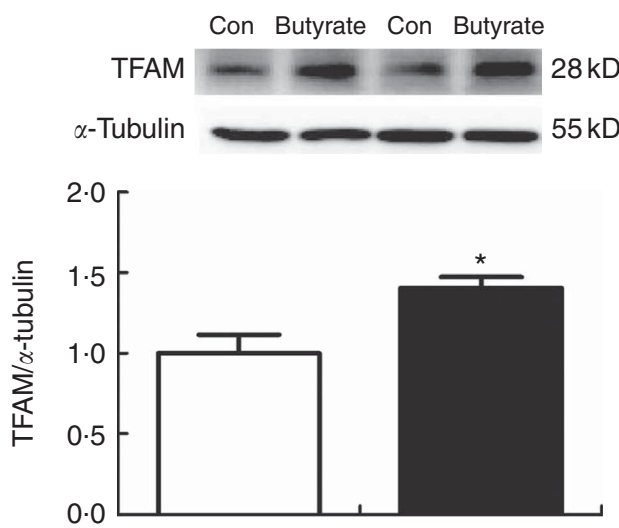

(c)
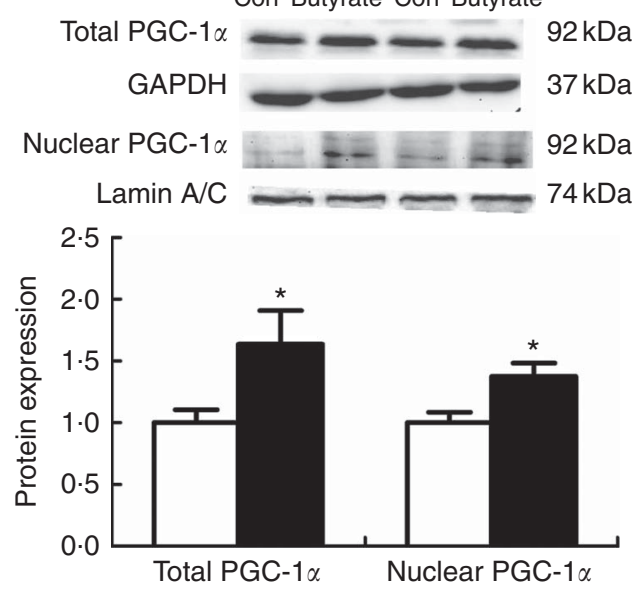

(b)
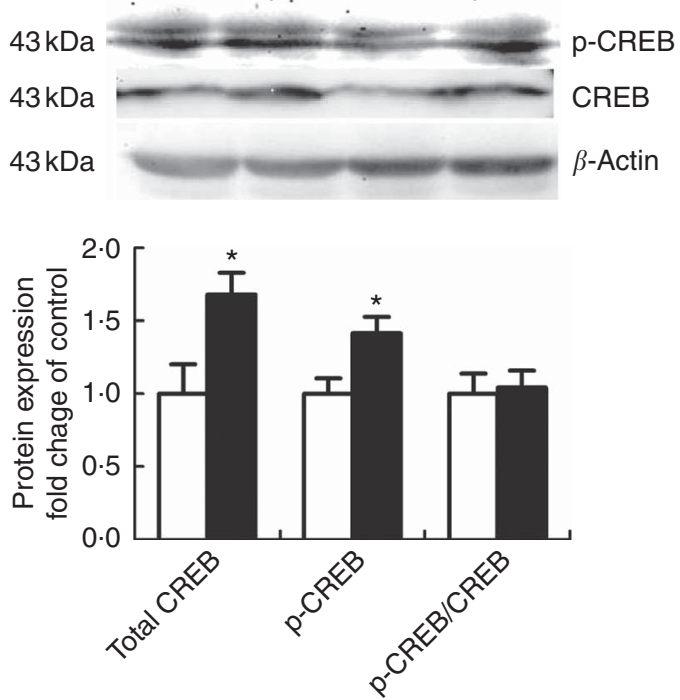

(d)

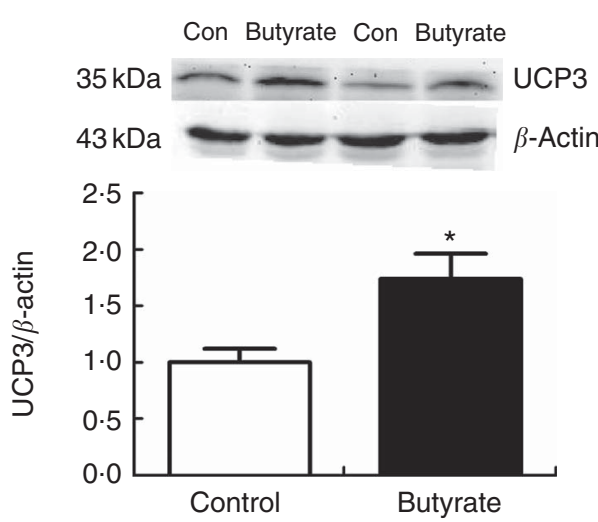

Fig. 3. Mitochondrial transcription factor A (TFAM) (a), phosphorylated cAMP response element-binding protein (CREB) and total CREB (b), total and nuclear PPARcoactivator-1 $a$ (PGC-1 $a)$ (c) and uncoupling protein 3 (UCP3) expressions (d) in gastrocnemius muscle of weaning rats ( $n 7$ per group). $\beta$-Actin, $\alpha$-tubulin, GAPDH and lamin $A / C$ were used as internal standards for Western blotting. Values are means with their standard errors. ${ }^{*} P<0.05$, compared with control. $\square$, Control; 口, butyrate: Con, control; p-CREB, anti-phospho-ser ${ }^{133}$ CREB.

group were significantly enhanced compared with control group (Fig. 3(c) and (d)).

\section{mRNA and protein expressions of myosin heavy chain in gastrocnemius muscle}

The type 1 myosin heavy chain (MYHC1) mRNA and protein expressions in the butyrate group were significantly higher than that of the control group $(P<0 \cdot 05)$. The mRNA expressions of type 2 variants, including MYHC2A, MYHC2B and MYHC2X, showed not significant changes between the two groups (Fig. 4). Similarly, mRNA expressions of MYOD (myogenic differentiation) and myogenin, myogenic regulatory factors and myoglobin did not change compared with the control group. The mRNA expression of mTOR (mechanistic target of rapamycin), which could regulate protein synthesis in muscle, was also not altered with maternal butyrate supplementation (Fig. 4).

\section{Discussion}

Numerous studies have shown that maternal nutrients influence the growth performance of their offspring ${ }^{(29,30)}$. Butyrate supplementation to gestating sows has been reported to enhance post-weaning growth performance of offspring ${ }^{(13)}$, although supplementation with sodium butyrate after farrowing did not have a significant effect on weaning body weight ${ }^{(31)}$. It has also been shown that butyrate supplementation of high-fat diet-fed mothers increased the number of fetuses compared with the control group ${ }^{(12)}$. Butyrate can act as a growth promoter in milk replacement formula for young calves ${ }^{(32)}$. Our previous study showed that maternal sodium butyrate supplementation elevated lipolysis in adipose tissue in dams and led to lipid metabolism changes in offspring liver of weaning-age rats $^{(28)}$. The present results show that maternal butyrate supplementation increased ATP content and EC in gastrocnemius muscle. This investigation provides evidence 


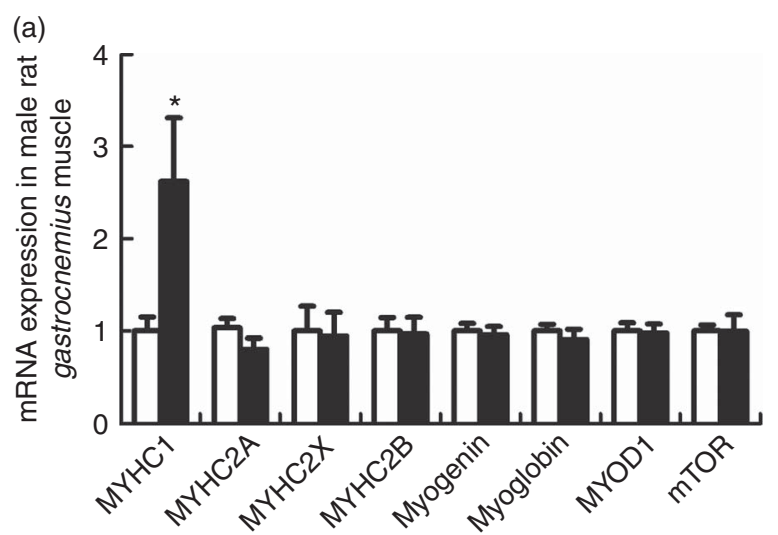

(b)
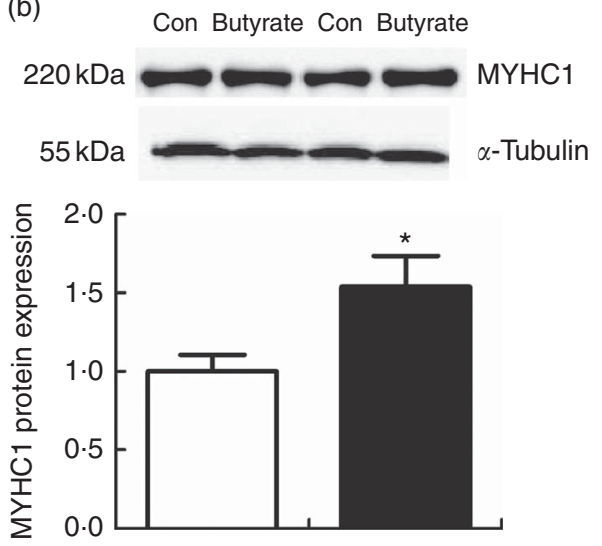

Fig. 4. Effect of maternal butyrate supplementation throughout gestation and lactation on mRNA expressions of the type of myosin heavy chain (MYHC) and MYOD, mTOR, myogenin and myoglobin in offspring gastrocnemius muscle ((a), $n 8$ per group); MYHC protein expression in offspring gastrocnemius muscle (b). $a$-Tubulin was used as the internal standard for Western blotting. Values are means with their standard errors. ${ }^{\star} P<0.05$, compared with control. $\square$, Control; $\square$, butyrate.

that maternal butyrate supplementation throughout gestation and lactation had effects on energy metabolism in offspring skeletal muscle.

Mitochondria use substrates and oxygen to produce ATP through the highly efficient process of OXPHOS in skeletal muscle. The biogenesis of mitochondria can prevent metabolic imbalances and promote healthy skeletal muscle ${ }^{(33,34)}$. In the present study, mitochondria-encoded genes such as COX3, ND5, ATP6 and ATP8 showed significant increases in offspring skeletal muscle of the butyrate group. In addition, COX1 and COX4 protein expressions also increased. Meanwhile, increased amount of mtDNA copy number was detected in offspring skeletal muscle of the butyrate group. The present results show that mitochondrial biogenesis was enhanced in offspring skeletal muscle with butyrate supplementation. The ATP demand required for muscle development is accommodated by elevations in mitochondrial biogenesis. TFAM is an important transcriptional activator of mitochondrial biogenesis, which allows for the nuclear control of mtDNA gene transcription and replication. As a previous study reported, accumulation of TFAM protein could increase the expressions of mtDNA and COX $1^{(35)}$. Our present results indicate that TFAM protein expression increased, which may enhance mitochondrial biogenesis by enhancing the expressions of mtDNA and COX1 in offspring skeletal muscle of the butyrate group.

We measured serum butyrate contents in both mother and offspring. The results showed that maternal serum butyrate content significantly increased in the treated group. The serum butyrate content in the offspring was too low to perform statistical analysis (data not shown). Several studies have established that through activation of their receptors, such as GPR43 and GPR41, SCFA regulate important signalling pathways involved in energy metabolism and homoeostasis ${ }^{(2,26)}$. In the present study, significant increases in GPR41 and GPR43 protein expressions were observed in offspring skeletal muscle of the butyrate group. The effect of butyrate on cAMP production and signalling pathway can be regulated by Gai-coupled GPR41 and GPR43 ${ }^{(36)}$. In ruminants, the SCFA could be acted as an external milk energy source. After birth, butyrate can be directly ingested by the neonates ${ }^{(37)}$. For butyrate crossing the placenta, previous studies have shown that multiple subtypes of maternal monocarboxylate transporters (MCT) were expressed ${ }^{(38)}$ in the placenta, and maternal high-fat diets with butyrate could increase MCT mRNA expression in the placenta ${ }^{(12)}$. Thus, our present study speculated that maternal butyrate supplementation during gestation and lactation could cross the placental barrier of dams and pass through milk of dams to offspring to influence offspring skeletal muscle metabolism. Further investigation will be needed to determine the effects of maternal butyrate on MCT expression in the placenta and the content in milk.

A previous study demonstrated that mitochondrial biogenesis could be promoted through activation of $\mathrm{p}$-CREB expression, accompanied by increased levels of $\mathrm{ATP}^{(39)}$. In the present study, an increase in both total and p-CREB content was observed in the treated group, although the ratio did not change. Further, we detected in offspring skeletal muscle, a significant increase in protein expression of PGC- $1 \alpha$, a major factor for the regulation of mitochondrial biogenesis ${ }^{(40)}$. CREB has been identified as an activator of PGC- $1 \alpha$ transcription by binding to the PGC- $1 \alpha$ promoter $^{(41)}$. Previous studies have demonstrated that $\mathrm{p}$-CREB mediates mitochondrial biogenesis probably through PGC- $1 \alpha^{(42,43)}$. The present results indicated that mitochondria biogenesis in offspring skeletal muscle might be mediated by PGC- $1 \alpha$ with enhanced GPR. Thus, we speculated that maternal butyrate supplementation enhanced mitochondrial biogenesis in offspring skeletal muscle by increasing the expressions of TFAM and COX and mtDNA copy number through the GPR and PGC- $1 \alpha$ pathways (Fig. 5).

As a mitochondrial transport protein, $\mathrm{UCP} 3$ was associated with the uncoupling of substrate oxidation ${ }^{(44-46)}$. Mitochondrial UCP3 was shown to play an important role in regulating the energy balance of skeletal muscle under fasting and high-fat feeding conditions ${ }^{(46)}$. In addition, a previous study suggested that UCP3 could be regulated by $\mathrm{p}$-CREB and participate in the regulation of fuel oxidation pathways ${ }^{(47)}$. Our study also demonstrated that maternal butyrate supplementation increased UCP3 protein expression in offspring skeletal muscle.

Differences in skeletal muscle fibre type are indicative of the oxidative capacity and mitochondrial content of skeletal muscle. Type 1 myofibres are highly oxidative and exhibit an 


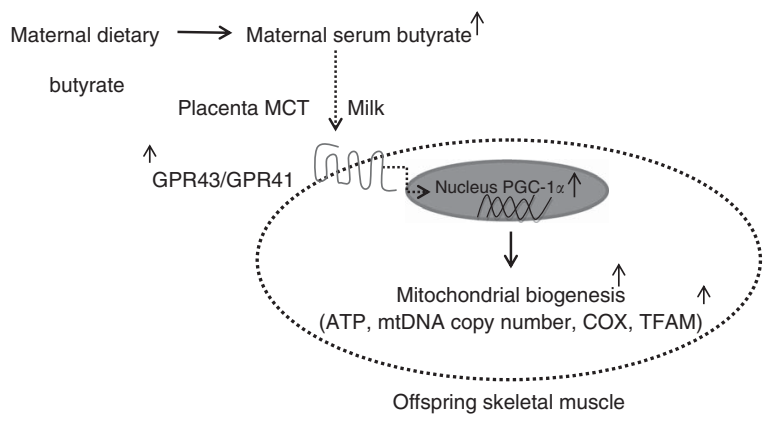

Fig. 5. Proposed mechanisms of maternal butyrate supplementation effect on mitochondrial biogenesis in offspring skeletal muscle. COX, cytochrome c oxidase; GPR, G-protein-coupled receptors; MCT, monocarboxylate transporters; mtDNA, mitochondrial DNA; PGC-1a, PPAR $y$-coactivator-1a; TFAM, mitochondrial transcription factor $A$.

increased number of mitochondria compared with type 2 fibres. It has been shown that butyrate supplementation in high-fat diets can promote type 1 fibre ratio in muscles associated with improved mitochondrial function ${ }^{(10,11)}$. In the present study, increased MYHC1 mRNA and protein expressions were observed in offspring skeletal muscles of the maternal butyrate group. The present study is the first to suggest that maternal butyrate supplementation could affect the myofibre type of offspring skeletal muscle.

The average daily food intake and weight gain of the dams showed no significant differences between the two groups. The body weight of weaning rats also did not change. The detected serum metabolic indices in mother and offspring showed no obvious changes, except for an increase in total cholesterol and LDL-cholesterol in offspring ${ }^{(28)}$. However, the data presented here show that maternal butyrate supplementation did not alter the expressions of MYOD, mTOR, myogenin and myoglobin in offspring skeletal muscle, which are important genes that regulate myofibre growth and differentiation. In our study, we added $1 \%$ butyrate into maternal diets of the butyrate group during gestation and lactation. We also added $4 \%$ alfalfa to the experimental diets of both groups. It could be digested and absorbed in the gastrointestinal tract by complete or partial fermentation to SCFA such as butyrate. Further investigations need to be carried out to determine the effects of the composition of the experiment diets without alfalfa on mitochondrial biogenesis.

In conclusion, the results of our study for the first time indicate that maternal butyrate supplementation during gestation and lactation significantly influence offspring skeletal muscle energy metabolism and mitochondria biogenesis at weaning. These effects may be mediated by the GPR and PGC- $1 \alpha$ pathways. However, follow-up studies are necessary to further clarify the detailed mechanisms on how this action is transferred through the placenta or milk to offspring from mothers supplemented with butyrate.

\section{Acknowledgements}

The authors gratefully acknowledge Dr Yue Li for her assistance in the animal experiment. This study was supported by the National Natural Science Foundation of China (31572482), the
National Key Research and Development Program of China (2016YFD0500502) and the Priority Academic Program Development of Jiangsu Higher Education Institutions.

The authors' contributions were as follows: all authors contributed to the study conception and design; Y. H. performed the experiments, analysed the data and wrote the draft manuscript; S. G. and G. J. maintained the animals; R. Z. and X. Y. provided scientific direction and interpreted the results; X. Y. revised and finalised the manuscript. All authors provided final approval of the manuscript. X. Y. is responsible for the integrity of the work as a whole.

The authors declare that they have no conflicts of interest.

\section{References}

1. Bergman EN (1990) Energy contributions of volatile fatty acids from the gastrointestinal tract in various species. Physiol Rev 70, 567-590

2. Macia L, Tan J, Vieira AT, et al. (2015) Metabolite-sensing receptors GPR43 and GPR109A facilitate dietary fibre-induced gut homeostasis through regulation of the inflammasome. Nat Commun 6, 6734 .

3. Anderson JW, Baird P, Davis RH, et al. (2009) Health benefits of dietary fiber. Nutr Rev 67, 188-205.

4. Lin Y, Zhuo Y, Fang ZF, et al. (2012) Effect of maternal dietary energy types on placenta nutrient transporter gene expressions and intrauterine fetal growth in rats. Nutrition $\mathbf{2 8}$, 1037-1043.

5. Yen JT, Nienaber JA, Hill DA, et al. (1991) Potential contribution of absorbed volatile fatty-acids to wholeanimal energy requirement in conscious swine. J Anim Sci 69, 2001-2012.

6. Sicilianojones J \& Murphy MR (1989) Production of volatile fatty-acids in the rumen and cecum-colon of steers as affected by forage - concentrate and forage physical form. J Dairy Sci 72, 485-492.

7. Meijer K, de Vos P \& Priebe MG (2010) Butyrate and other short-chain fatty acids as modulators of immunity: what relevance for health? Curr Opin Clin Nutr Metab Care 13, $715-721$.

8. Gao ZG, Yin J, Zhang J, et al. (2009) Butyrate improves insulin sensitivity and increases energy expenditure in mice. Diabetes 58, 1509-1517.

9. Conterno L, Fava F, Viola R, et al. (2011) Obesity and the gut microbiota: does up-regulating colonic fermentation protect against obesity and metabolic disease? Genes Nutr 6, 241-260.

10. Henagan TM, Stefanska B, Fang ZD, et al. (2015) Sodium butyrate epigenetically modulates high-fat diet-induced skeletal muscle mitochondrial adaptation, obesity and insulin resistance through nucleosome positioning. Br J Pharmacol 172, 2782-2798.

11. Gao Z, Yin J, Zhang J, et al. (2009) Butyrate improves insulin sensitivity and increases energy expenditure in mice. Diabetes 58, 1509-1517.

12. Lin Y, Fang ZF, Che LQ, et al. (2014) Use of sodium butyrate as an alternative to dietary fiber: effects on the embryonic development and anti-oxidative capacity of rats. PLOS ONE 9, e97838.

13. Lu H, Su S \& Ajuwon KM (2012) Butyrate supplementation to gestating sows and piglets induces muscle and adipose tissue oxidative genes and improves growth performance. I Anim Sci $\mathbf{4}, 430-432$. 
14. Scheffler TL, Scheffler JM, Park S, et al. (2014) Fiber hypertrophy and increased oxidative capacity can occur simultaneously in pig glycolytic skeletal muscle. Am J Physiol Cell Physiol 306, 354-363.

15. Wenz T, Diaz F, Spiegelman BM, et al. (2008) Activation of the PPAR/PGC-1alpha pathway prevents a bioenergetic deficit and effectively improves a mitochondrial myopathy phenotype. Cell Metab 8, 249-256.

16. Komen JC \& Thorburn DR (2014) Turn up the power pharmacological activation of mitochondrial biogenesis in mouse models. Br J Pharmacol 171, 1818-1836.

17. Baar K (2004) Involvement of PPAR gamma co-activator-1, nuclear respiratory factors 1 and 2, and PPAR alpha in the adaptive response to endurance exercise. Proc Nutr Soc 63 , 269-273.

18. Miura S, Kai Y, Ono M, et al. (2003) Overexpression of peroxisome proliferator-activated receptor gamma coactivator1alpha down-regulates GLUT4 mRNA in skeletal muscles. J Biol Chem 278, 31385-31390.

19. Lin J, Wu H, Tarr PT, et al. (2002) Transcriptional co-activator PGC-1 alpha drives the formation of slow-twitch muscle fibres. Nature 418, 797-801.

20. Than TA, Lou H, Ji C, et al. (2011) Role of cAMP-responsive element-binding protein (CREB)-regulated transcription coactivator 3 (CRTC3) in the initiation of mitochondrial biogenesis and stress response in liver cells. J Biol Chem 286, 22047-22054.

21. Inoue D, Kimura I, Wakabayashi $M$, et al. (2012) Short-chain fatty acid receptor GPR41-mediated activation of sympathetic neurons involves synapsin $2 \mathrm{~b}$ phosphorylation. FEBS Lett 586, 1547-1554.

22. Samuel BS, Shaito A, Motoike T, et al. (2008) Effects of the gut microbiota on host adiposity are modulated by the short-chain fatty-acid binding $G$ protein-coupled receptor, Gpr41. Proc Natl Acad Sci U S A 105, 16767-16772.

23. Hu J, Kyrou I, Tan BK, et al. (2016) Short-chain fatty acid acetate stimulates adipogenesis and mitochondrial biogenesis via GPR43 in brown adipocytes. Endocrinology 157, 1881-1894.

24. Zhao GH, Liu JF, Nyman M, et al. (2007) Determination of short-chain fatty acids in serum by hollow fiber supported liquid membrane extraction coupled with gas chromatography. J Chromatogr B 846, 202-208.

25. Liu X, Wang J, Li R, et al. (2011) Maternal dietary protein affects transcriptional regulation of myostatin gene distinctively at weaning and finishing stages in skeletal muscle of Meishan pigs. Epigenetics 6, 899-907.

26. Jia Y, Li R, Cong R, et al. (2013) Maternal low-protein diet affects epigenetic regulation of hepatic mitochondrial DNA transcription in a sex-specific manner in newborn piglets associated with GR binding to its promoter. PLOS ONE $\mathbf{8}$, e63855.

27. Jia LF, Li J, He B, et al. (2016) Abnormally activated onecarbon metabolic pathway is associated with mtDNA hypermethylation and mitochondrial malfunction in the oocytes of polycystic gilt ovaries. Sci Rep 6, 19436.

28. Zhou JB, Gao SX, Chen JL, et al. (2016) Maternal sodium butyrate supplement elevates the lipolysis in adipose tissue and leads to lipid accumulation in offspring liver of weaningage rats. Lipids Health Dis 15, 119.

29. Pantaleao LC, Teodoro GF, Torres-Leal FL, et al. (2013) Maternal postnatal high-fat diet, rather than gestational diet, affects morphology and mTOR pathway in skeletal muscle of weaning rat. J Nutr Biochem 24, 1340-1348.

30. Max D, Brandsch C, Schumann S, et al. (2014) Maternal vitamin D deficiency causes smaller muscle fibers and altered transcript levels of genes involved in protein degradation, myogenesis, and cytoskeleton organization in the newborn rat. Mol Nutr Food Res 58, 343-352.
31. Wang J, Yang M, Xu S, et al. (2014) Comparative effects of sodium butyrate and flavors on feed intake of lactating sows and growth performance of piglets. Anim Sci J 85, 683-689.

32. Guilloteau P, Zabielski R, David JC, et al. (2009) Sodiumbutyrate as a growth promoter in milk replacer formula for young calves. J Dairy Sci $\mathbf{9 2}, 1038-1049$.

33. Patti ME, Butte AJ, Crunkhorn S, et al. (2003) Coordinated reduction of genes of oxidative metabolism in humans with insulin resistance and diabetes: potential role of PGC1 and NRF1. Proc Natl Acad Sci U S A 100, 8466-8471.

34. Joseph AM, Joanisse DR, Baillot RG, et al. (2012) Mitochondrial dysregulation in the pathogenesis of diabetes: potential for mitochondrial biogenesis-mediated interventions. Exp Diabetes Res 2012, 642038.

35. Wen S, Gao J, Zhang L, et al. (2016) p53 increase mitochondrial copy number via up-regulation of mitochondrial transcription factor $\mathrm{A}$ in colorectal cancer. Oncotarget (epublication ahead of print version 7 October 2016).

36. Le Poul E, Loison C, Struyf S, et al. (2003) Functional characterization of human receptors for short chain fatty acids and their role in polymorphonuclear cell activation. $J$ Biol Chem 278, 25481-25489.

37. Castro JJ, Gomez A, White B, et al. (2016) Changes in the intestinal bacterial community, short-chain fatty acid profile, and intestinal development of preweaned Holstein calves. 2. Effects of gastrointestinal site and age. J Dairy Sci 99, 9703-9715.

38. Wanaga T \& Kishimoto A (2015) Cellular distributions of monocarboxylate transporters: a review. Biomed Res 36, 279-301.

39. Zuo LN, Li Q, Sun B, et al. (2013) Cilostazol promotes mitochondrial biogenesis in human umbilical vein endothelial cells through activating the expression of PGC-1 alpha. Biochem Biophys Res Commun 433, 52-57.

40. Gleyzer N, Vercauteren K \& Scarpulla RC (2005) Control of mitochondrial transcription specificity factors (TFB1M and TFB2M) by nuclear respiratory factors (NRF-1 and NRF-2) and PGC-1 family coactivators. Mol Cell Biol 25, 1354-1366.

41. Chowanadisai W, Bauerly KA, Tchaparian E, et al. (2010) Pyrroloquinoline quinone stimulates mitochondrial biogenesis through CAMP response element-binding protein phosphorylation and increased PGC-1 alpha expression. $J$ Biol Chem 285, 142-152.

42. Mehta SL, Mendelev N, Kumari S, et al. (2013) Overexpression of human selenoprotein $\mathrm{H}$ in neuronal cells enhances mitochondrial biogenesis and function through activation of protein kinase $\mathrm{A}$, protein kinase $\mathrm{B}$, and cyclic adenosine monophosphate response element-binding protein pathway. Int J Biochem Cell Biol 45, 604-611.

43. Kim HK, Song IS, Lee SY, et al. (2014) B7-H4 downregulation induces mitochondrial dysfunction and enhances doxorubicin sensitivity via the cAMP/CREB/PGC1-alpha signaling pathway in HeLa cells. Pflugers Arch 466, 2323-2338.

44. Boss O, Hagen T \& Lowell BB (2000) Uncoupling proteins 2 and 3: potential regulators of mitochondrial energy metabolism. Diabetes 49, 143-156.

45. Gong DW, He Y, Karas M, et al. (1997) Uncoupling protein-3 is a mediator of thermogenesis regulated by thyroid hormone, beta3-adrenergic agonists, and leptin. J Biol Chem 272, 24129-24132.

46. Nabben M \& Hoeks J (2008) Mitochondrial uncoupling protein 3 and its role in cardiac- and skeletal muscle metabolism. Physiol Behav 94, 259-269.

47. Dorsa KK, dos Santos MV \& da Silva MRD (2010) Enhancing T3 and CAMP responsive gene participation in the thermogenic regulation of fuel oxidation pathways. Arq Bras Endocrinol Metabol 54, 381-389. 